\title{
A GENERALIZATION OF A RESULT OF SINNOTT
}

\author{
H. KISILEVSKY
}

To the memory of Olga Taussky-Todd, a friend, a collegue and an inspiration

\section{Sinnott's Theorem.}

Let $p$ be a prime number and suppose that $\Gamma$ is a pro- $p$-group isomorphic to $\mathbb{Z}_{p}$, the additive group of $p$-adic integers. For each integer $n$, let $\Gamma_{n}=p^{n} \Gamma$ and $G_{n}=\Gamma / \Gamma_{n}$.

Let $A$ be a discrete $\Gamma$-module and define

$$
A_{n}=A^{\Gamma_{n}}=\left\{a \in A \mid \gamma(a)=a \text { for all } \gamma \in \Gamma_{n}\right\} .
$$

Then $A=\cup A_{n}$.

Proposition 1. If $A_{n}$ is finite for all $n$, then

$$
\left|A_{n+1}\right| \equiv\left|A_{n}\right| \quad\left(\bmod p^{n+1}\right)
$$

Proof. $A_{n+1}$ is a finite $G_{n+1}$-module so that

$$
A_{n+1}=B \cup C
$$

where $B$ is the set of those elements in $A_{n+1}$ not fixed by any non-trivial element of $G_{n+1}$, and $C=A_{n+1} \backslash B$. Since $G_{n+1}$ is a cyclic group it follows that every element of $C$ is fixed by the the subgroup of order $p$ in $G_{n+1}$, and so $C \subseteq A^{\Gamma_{n}}=A_{n}$. The opposite inclusion is clear so $C=A_{n}$. Counting we have,

$$
\left|A_{n+1}\right|=|B|+\left|A_{n}\right| .
$$

Since $B$ is a union of orbits each of which contains $p^{n+1}$ elements it follows that

$$
\left|A_{n+1}\right| \equiv\left|A_{n}\right| \quad\left(\bmod p^{n+1}\right)
$$


Corollary 1. If $A_{n}$ is finite for all $n$, then $\lim _{n \rightarrow \infty}\left|A_{n}\right|$ exists p-adically.

Proof. It follows from Proposition 1 that for all $m \geq n$

$$
\left|A_{m}\right| \equiv\left|A_{n}\right| \quad\left(\bmod p^{n+1}\right) \text {. }
$$

Hence the sequence $\left\{\left|A_{n}\right|\right\}_{n=1,2, \ldots}$ is a $p$-adic Cauchy sequence and therefore has a $p$-adic limit.

Let $k_{\infty} / k$ be a $\mathbb{Z}_{p}$-extension of number fields (resp. function fields over finite fields) and denote by $C_{n}$ the ideal class group (resp. the group of divisor classes of degree zero) of the $n^{\text {th }}$-layer $k_{n}$ of $k_{\infty} / k$. For any set $S$ of prime numbers, finite or infinite, let $C_{n}(S)$ be the largest subgroup of $C_{n}$ whose order is divisible only by primes in $S$. Let

$$
C(S)=\lim _{\rightarrow} C_{n}(S)
$$

be the direct limit with respect to the natural maps induced by extension of ideals.

We obtain the following generalization of a result of Sinnott (proved in the case of a cyclotomic $\mathbb{Z}_{p}$-extension of a CM ground field and for the "minus" class number).

Corollary 2 (Sinnott $[\mathbf{S}]$ ). Let $k_{\infty} / k$ be a $\mathbb{Z}_{p}$-extension of the global field $k$. For any set of prime numbers $S$, if $p \notin S$, then $\lim _{n \rightarrow \infty}\left|C_{n}(S)\right|$ exists p-adically.

Proof. Since $p \notin S$ it follows that for $n \leq m$, the natural map $C_{n}(S) \longrightarrow$ $C_{m}(S)$ is an injection. Then $C(S)=\cup C_{n}(S)$ and

$$
C_{n}(S)=C(S)^{\Gamma_{n}} \text {. }
$$

The result then follows from Proposition 1.

Remark. It is a consequence of Iwasawa theory that $\lim _{n \rightarrow \infty}\left|C_{n}(S)\right|$ exists $p$-adically for any set $S$ of primes.

Corollary 3 (Washington $[\mathbf{W}]$ ). Let $k_{\infty} / k$ be a $\mathbb{Z}_{p}$-extension of global fields and let $l$ be a prime $l \neq p$. Take $S=\{l\}$ so that $C_{n}(S)$ is the l-primary part of the class group of $k_{n}$. If $\left|C_{n}(S)\right|=l^{a_{n}}$, then either $\left\{a_{n}\right\}$ is eventually constant or else there is a constant $c$, independent of $n$ such that $a_{n} \geq c p^{n}$ for infinitely many $n$.

Proof. It follows from Proposition 1 that

$$
l^{a_{n+1}} \equiv l^{a_{n}} \quad\left(\bmod p^{n+1}\right)
$$

and therefore $a_{n+1}-a_{n}$ is divisible by the order of $l\left(\bmod p^{n+1}\right)$. For large $n$ this order is $c p^{n}$ for some constant $c$ (depending only on $l$ ) hence either $\left\{a_{n}\right\}$ is eventually constant or else $a_{n} \geq c p^{n}$ for infinitely many $n$. 


\section{Function fields.}

Let $k_{\infty} / k$ be the constant $\mathbb{Z}_{p}$-extension of the function field $k$, and let $h_{n}$ be the number of divisor classes of degree zero (the class number) of the $n^{\text {th }}$ layer $k_{n}$. Denote by $h_{n}^{\prime}$ the "prime-to- $p$ " part of $h_{n}$ so that $h_{n}^{\prime}=h_{n} / p^{e_{n}}=h_{n}(S)$ where $S$ is the set of all primes other than $p$, and $e_{n}$ is the largest power of $p$ in $h_{n}$. Then $h_{n}$ is given by

$$
h_{n}=\prod_{i=1}^{2 g}\left(1-\alpha_{i}^{p^{n}}\right)=p^{e_{n}} h_{n}^{\prime}
$$

where

$$
\zeta_{k}(s)=Z_{k}(t)=\frac{\prod\left(1-\alpha_{i} t\right)}{(1-t)(1-q t)}
$$

is the $\zeta$-function of $k, q=p^{f}$ is the order of the finite field of $k$, and $t=q^{-s}$. The numbers $\alpha_{i}$ are algebraic integers with $\alpha_{i} \bar{\alpha}_{i}=q$.

Let $K=\mathbb{Q}\left(\alpha_{1}, \ldots, \alpha_{2 g}\right)$ be the field generated over the rational field $\mathbb{Q}$ by the reciprocal roots of $Z_{k}(t)$, and fix $\mathcal{P}$ a prime ideal of $K$ dividing $p$. We compute the $\mathcal{P}$-adic $\operatorname{limit}_{n \rightarrow \infty} \lim _{n}^{\prime}$ in a completion $K_{\mathcal{P}}$ of $K$. Note that if $\alpha_{i}$ is not a $\mathcal{P}$-unit then

$$
\lim _{n \rightarrow \infty}\left(1-\alpha_{i}^{p^{n}}\right)=1
$$

and hence

$$
\lim _{n \rightarrow \infty} h_{n}^{\prime}=\lim _{n \rightarrow \infty} h_{n} / p^{e_{n}}=\lim _{n \rightarrow \infty} p^{-e_{n}} \prod^{\circ}\left(1-\alpha_{i}^{p^{n}}\right)
$$

where the product is taken over those $i$ such that $\alpha_{i}$ is a $\mathcal{P}$-unit. Observe also that if $\alpha$ is a 1-unit, (i.e., if $\alpha$ is congruent to $1 \bmod \mathcal{P}$ ), then we can define $u_{n}$ by the equation

$$
\alpha^{p^{n}}=1+p^{n} u_{n}
$$

Taking $p$-adic logarithms we find,

$$
p^{n} \log _{p} \alpha=p^{n} u_{n}-p^{2 n} u_{n}^{2} / 2+\cdots
$$

so

$$
u_{n} \equiv \log _{p} \alpha \quad\left(\bmod p^{n}\right)
$$

Now

$$
p^{-e_{n}} \prod^{\circ}\left(1-\alpha_{i}^{p^{n}}\right)=p^{-e_{n}} \prod^{\prime}\left(1-\alpha_{i}^{p^{n}}\right) \cdot \prod^{\prime \prime}\left(1-\alpha_{i}^{p^{n}}\right)
$$

where the product $\prod^{\prime}$ is taken over those $i$ such that $\alpha_{i}$ is a 1-unit, and $\prod^{\prime \prime}$ is taken over those $i$ such that $\alpha_{i}$ is a $\mathcal{P}$-unit but not a 1 -unit. Let $\nu_{i} \in \mathbb{Q}$ 
be the $p$-adic valuation of $\log \alpha_{i}$. Let $\lambda$ be the number of $i$ such that $\alpha_{i}$ is a 1-unit. Since $h_{n}^{\prime}$ is prime to $p$, it follows that

$$
e_{n}=\lambda n+\sum_{i=1}^{\lambda} \nu_{i}
$$

for all sufficiently large $n$, and that

$$
\lim _{n \rightarrow \infty} p^{-e_{n}} \prod^{\prime}\left(1-\alpha_{i}^{p^{n}}\right)=(-1)^{\lambda} \frac{\prod^{\prime} \log _{p} \alpha_{i}}{p^{\sum \nu_{i}} .}
$$

Since $\lim _{n \rightarrow \infty} h_{n}^{\prime}$ and $\lim _{n \rightarrow \infty} p^{-e_{n}} \prod^{\prime}\left(1-\alpha_{i}^{p^{n}}\right)$ exist $p$-adically, it follows that

$$
\lim _{n \rightarrow \infty} \prod^{\prime \prime}\left(1-\alpha_{i}^{p^{n}}\right)
$$

exists.

For any unit $\beta \in K_{\mathcal{P}}$

$$
\beta=\omega(\beta) \cdot\langle\beta\rangle
$$

where $\omega(\beta)$ is a root of unity of order dividing $p^{r}-1$ with $p^{r}=N_{K / \mathbb{Q}}(\mathcal{P})$ and $\langle\beta\rangle$ a 1-unit. It is then clear that

$$
\omega(\beta)=\lim _{n \rightarrow \infty} \beta^{p^{r n}} .
$$

Since the limit $\lim _{n \rightarrow \infty} \prod^{\prime \prime}\left(1-\alpha_{i}^{p^{n}}\right)$ exists, it can be computed by letting $n$ run through multiples of $r$, and we obtain the following:

Proposition 2. For the constant $\mathbb{Z}_{p}$-extension of function fields we have

$$
\lim _{n \rightarrow \infty} h_{n}^{\prime}=(-1)^{\lambda} \cdot \frac{\prod^{\prime} \log _{p} \alpha_{i}}{p^{\sum \nu_{i}}} \cdot \prod^{\prime \prime}\left(1-\omega\left(\alpha_{i}\right)\right) .
$$

Corollary 4. For the constant $\mathbb{Z}_{p}$-extension of function fields and for any integer $j$,

$$
\prod^{\prime \prime}\left(1-\omega\left(\alpha_{i}\right)\right)=\prod^{\prime \prime}\left(1-\omega^{j}\left(\alpha_{i}\right)\right)
$$

This can be proved directly using the fact that $Z_{k}(t)$ is a rational function in $\mathbb{Q}(t)$.

\section{References}

[S] W. Sinnott, Talk given at Iwasawa Conference, MSRI, 1985. 
[W] L.C. Washington, The non-p-part of the class number in a cyclotomic $\mathbb{Z}_{p}$-extension, Invent. Math., 49(1) (1978), 87-97.

This work was supported in part by grants from NSERC and FCAR.

CONCORDIA UNIVERSITY

1455 de Maisonneuve Blvd. West

Montréal, Quebec, H3G 1M8, CANADA

E-mail address: kisilev@cicma.concordia.ca 